\title{
DIVERSIDAD SEXUAL Y DE GÉNERO EN EL MARCO DEL CONFLICTO ARMADO EN COLOMBIA. ALGUNAS REFLEXIONES PARA SU ESTUDIO.*
}

\author{
SEXUAL AND GENDER DIVERSITY IN THE FRAMEWORK OF THE ARMED \\ CONFLICT IN COLOMBIA. SOME REFLECTIONS FOR ITS STUDY
}

Sebastí́n Giraldo-AguirRE**

\begin{abstract}
Resumen
Objetivo. Presentar algunas reflexiones conceptuales, metodológicas y políticas para el estudio de la diversidad sexual y de género en el marco del conflicto armado y en el proceso de construcción de paz. Metodología. Para plantear estas consideraciones se realizó una búsqueda bibliográfica en bases de datos de internet y una revisión de prensa para identificar las apuestas académicas, gubernamentales y periodísticas que se han desarrollado al respecto. Resultado. Debido a que es un campo de estudio reciente en el país, se requieren algunas advertencias preliminares que busquen enriquecer la apuesta investigativa sobre el tema. Conclusión. La implementación de la perspectiva de género y, en específico, de la perspectiva de diversidad sexual y de género, son mecanismos fundamentales para el proceso transicional que afronta Colombia.
\end{abstract}

Palabras clave: diversidad sexual, conflicto armado, guerra, género, paz, Colombia.

\begin{abstract}
Objective. To present some conceptual, methodological and political reflections for the study of sexual and gender diversity in the context of the armed conflict and in the process of peace building in the country. Methodology. To propose these considerations a bibliographic search in databases and a newspaper review was carried out to identify the academic, government and journalistic documents that have been developed in this regard. Results. Because it is a recent field of study in the country, some preliminary warnings that seek to enrich the research on the subject are needed. Conclusion. The implementation of the gender perspective and, specifically that of the sexual diversity and gender perspective are fundamental mechanisms for the transitional process that Colombia is facing.
\end{abstract}

Key words: sexual diversity, armed conflict, war, gender, peace, Colombia.

\footnotetext{
* La primera versión de este escrito se presentó en el XXIII Coloquio Internacional de Estudios de Género Sexualidad y Estado, organizado por el Programa Universitario de Estudios de Género de la Universidad Nacional Autónoma de México -UNAM-, que se llevó a cabo el 3 y 4 de noviembre de 2016.

** Universidad de Caldas. Manizales, Colombia. E-mail: sebastian.giraldo@ucaldas.edu.co -

(D) orcid.org/0000-0003-4207-2806 Google Scholar
} 
Diversidad sexual y de género en el marco del conflicto armado en colombia. Algunas reflexiones para su estudio.

\section{Introducción}

La investigación sobre diversidad sexual y de género en contextos de violencia armada tiene pocos antecedentes en el país y se podría decir que en el mundo. Una de las razones de esta condición fue la carencia hasta la década de 1990 de la perspectiva de género en los estudios sobre conflictos y paz (Goldstein, 2003; Serrano, 2013). Este antecedente ha tenido varias repercusiones, entre ellas la baja consideración de la relación entre diversidad sexual, guerra y construcción de paz en este campo de estudios (Serrano, 2004).

Siguiendo al autor, este panorama cambió gracias a la Conferencia de Beijing (1995) y a la Resolución 1325 de las Naciones Unidas (ONU, 2000), las cuales suscitaron el reconocimiento del impacto de los conflictos en la vida de las mujeres. Por tal razón, se emprendió una incorporación más firme de la perspectiva de género, tanto en los análisis de los conflictos armados como en las labores de las organizaciones encargadas de la construcción de paz (Serrano, 2013, p. 54). En medio de esta apuesta institucional, la agenda "LGBT" encontró un terreno propicio para su emergencia y su consolidación.

En etapas previas a dicho periodo, se habían documentado varios tipos de violencias por razones de diversidad sexual y de género en contextos bélicos. Casos como la persecución en la Alemania nazi, en el régimen franquista de España, en el apartheid de Sudáfrica, y los conflictos en Europa del este, Perú y El Salvador son algunos ejemplos (Theidon, 2004; Albarracín y Rincón, 2013; Palevi, 2016), incluso en algunos de ellos han sido reconocidas las víctimas "LGBT". Sin embargo, fueron episodios que contaron con un menor apoyo institucional e internacional para su gestión. Luego de la puesta en marcha de las plataformas que introdujeron la perspectiva de género, se ha fortalecido el proceso de reconocimiento y atención a este grupo de víctimas. La figura de los refugiados LGBT en los conflictos de Medio Oriente o de África, y la categoría de víctimas LGBT en Colombia son una evidencia de ello. Frente al tema, por ejemplo, algunas cifras de la Unidad de Víctimas demuestran la transcendencia del tema:

Tabla 1. Víctimas del conflicto armado en Colombia. Enfoque diferencial: género.

\begin{tabular}{lr}
\hline Género & \multicolumn{1}{c}{ Personas } \\
\hline Mujer & 4.051 .692 \\
Hombre & 4.048 .209 \\
No informa & 59.120 \\
LGBTI & 1.966 \\
\hline
\end{tabular}

Fuente: RUV. Registro Único de Víctimas, agosto de 2017. Recuperado de https://rni.unidadvictimas.gov.co/RUV 
En este sentido, el caso colombiano se constituye en un escenario que permite intersectar con mayor robustez estas dos aristas de investigación: el conflicto armado y la diversidad sexual y de género. En otras confrontaciones bélicas, como las citadas renglones atrás, los análisis fueron escasos y posteriores a los procesos de negociación de paz, debido a que la agenda "LGBT" todavía estaba desterrada del ámbito político de aquellos Estados. Este antecedente es distinto para Colombia, pues el proceso de politización de esta materia ha coexistido con la permanencia del conflicto armado y con los procesos de negociación de paz, lo cual permite plantear otras perspectivas para analizarlos.

Por lo tanto, la coyuntura política que atraviesa el país se convierte en una oportunidad para reflexionar las implicaciones teóricas, metodológicas y políticas que trae consigo el análisis de la diversidad sexual y de género en contextos de violencia política, sobre sus repercusiones para la justicia transicional y para la construcción de paz. El presente artículo intenta emprender esta tarea. Para ello, en un primer momento, se realiza un breve recuento sobre la emergencia de la agenda "LGBT" en el marco del conflicto armado en el país. Posteriormente, se plantean una serie de reflexiones que pretenden señalar algunas advertencias para el estudio de la diversidad sexual y de género en contextos de guerra como el colombiano. Por último, a manera de conclusión, se realizan algunas consideraciones sobre la justicia transicional.

\section{Discusión}

\section{La emergencia de la agenda "LGBT" relacionada con el conflicto armado colombiano}

A pesar de que el país ha estado en medio del conflicto armado por más de 50 años, la preocupación por la perspectiva de la diversidad sexual y de género en este ámbito es reciente. La puesta en marcha de dicho enfoque obedece a varias etapas en las cuales han estado involucrados distintos actores. En orden cronológico, las organizaciones sociales en defensa de los derechos humanos fueron las primeras en advertir esta situación. Luego, el Estado por medio de varias dependencias atendió este llamado y, posteriormente, los medios de comunicación comenzaron a incluirlo en sus notas. Durante este proceso, la academia lentamente ha venido consolidándolo como un nuevo campo de estudios sobre la violencia armada en el país.

El origen de la incorporación de la perspectiva de género en el marco del conflicto armado se remonta a los años 80 del siglo pasado, cuando algunos movimientos feministas comenzaron a denunciar los efectos diferenciales de la guerra en los cuerpos de las mujeres (Wills y Gómez, 2006). Dicha perspectiva se consolida con mayor ímpetu en la década posterior debido a las exhortaciones dirigidas al Estado colombiano por diferentes instancias nacionales e internacionales. En primer lugar, la Constitución Política de 1991 posicionó el discurso 
Diversidad sexual y de género en el marco del conflicto armado en colombia. Algunas reflexiones para su estudio.

referido a la necesidad de incluir perspectivas diferenciales en la implementación de políticas públicas que atendieran asuntos referentes a la discriminación, exclusión y violencias marcadas por diferencias de género, edad, pertenencia étnica, discapacidad y pobreza (Centro Nacional de Memoria Histórica-CNMH-, 2013, p. 35). En el ámbito internacional, la Convención Belém do Pará (1994) - Convención Interamericana para Prevenir, Sancionar y Erradicar la Violencia contra la Mujer-, la Conferencia de Beijing (1995) -IV Conferencia Mundial sobre la Mujer- y la Resolución 1325 de las Naciones Unidas fueron plataformas fundamentales para impulsar programas gubernamentales que atendieran la victimización de las mujeres en contextos de violencia política. Sin embargo, hasta este periodo la perspectiva de género se dirigía primordialmente a la situación de las mujeres.

Por su parte, los asuntos de diversidad sexual y de género, a pesar de que empezaron a ser documentados desde la década de 1990 por organismos internacionales (Ordóñez, 1996), tomaron mayor relevancia a comienzos de la década posterior, la cual fue, justamente, una etapa fundamental para la institucionalización de la agenda "LGBT" en el país. Este proceso se evidencia en la conformación de organizaciones sociales y en los reconocimientos jurídicos por parte de la Corte Constitucional. Aquellas organizaciones son, precisamente, las que desde su nacimiento emprenden la tarea de denunciar la victimización diferencial de esta población en el marco del conflicto armado. En palabras de Albarracín y Rincón (2013), el movimiento LGBTI replicó las estrategias de los grupos de derechos humanos con el fin de dignificar a sus muertos y producir un cambio institucional que impactara en la prevención, investigación y sanción de estos hechos.

La Corporación Planeta Paz, que fue una iniciativa creada durante los diálogos entre el Gobierno de Andrés Pastrana y las FARC-EP, fue uno de los primeros colectivos en señalar las afectaciones que recibían lesbianas, gais y personas transgénero en los territorios donde se concentraban las confrontaciones. Posteriormente, se suman a esta labor Colombia Diversa en el 2004, Santamaría Fundación en el 2005 y Caribe Afirmativo en el 2009. Con el paso del tiempo, los informes de derechos humanos realizados por estas organizaciones cada vez fueron integrando más entre sus páginas la situación de las víctimas LGBT del conflicto armado.

Como respuesta a los señalamientos de aquellos informes, el Estado colombiano comenzó a reconocer la perspectiva de diversidad sexual y de género en los programas de atención a las víctimas del conflicto armado. Algunas entidades creadas en el marco de la Ley de Justicia y Paz (Ley 975 de 2005), como la Comisión Nacional de Reparación y Reconciliación, protagonizaron esta apuesta institucional. Algunas gestiones promovidas al respecto fueron: la introducción de la orientación sexual en las planillas de registro, la puesta en marcha de protocolos de atención especiales y la promoción a la denuncia.

Posteriormente, en una apuesta estatal distinta, la Ley de Víctimas y Restitución de Tierras (Ley 1448 de 2011) consolida la atención diferencial de los sectores inmersos en el conjunto 
de las víctimas, entre ellos la población LGBT. Esta perspectiva se reafirma gracias a que su articulado establece:

\begin{abstract}
Artículo 13. Enfoque diferencial: El principio de enfoque diferencial reconoce que hay poblaciones con características particulares en razón de su edad, género, orientación sexual y situación de discapacidad. Por tal razón, las medidas de ayuda humanitaria, atención, asistencia y reparación integral que se establecen en la presente Ley, contarán con dicho enfoque. (Ley 1448 de 2011)
\end{abstract}

Como se puede apreciar, el género y la orientación sexual se consideran dentro de este abanico de categorías, lo que garantiza la puesta en marcha de un marco institucional para su administración. Bajo esta plataforma se crean entidades como la Unidad para la Atención y Reparación Integral a las Víctimas (Unidad de Víctimas), que tiene a su cargo la creación e implementación de la política pública de atención y reparación a víctimas, y el Centro Nacional de Memoria Histórica (CNMH), que se encarga de promover procesos que garanticen el derecho de las víctimas y de la sociedad en su conjunto a la reconstrucción de la memoria histórica. Estas instancias son las líderes en instaurar aquellos enfoques. Como ejemplo de ello, se encuentran las publicaciones preliminares de los informes "Víctimas del conflicto armado interno con orientaciones sexuales diversas" (Unidad para la atención y reparación integral a las víctimas, 2014) y "Enfoque de orientaciones sexuales e identidades de género" (USAID, OIM y Unidad para la atención y reparación integral a las víctimas, 2015), al igual que el informe "Aniquilar la diferencia. Lesbianas, gays, bisexuales y transgeneristas en el marco del conflicto armado colombiano" (CNMH, 2015), el cual es un ejercicio testimonial con pocos antecedentes en el país y en el mundo.

Simultáneamente, durante la primera mitad de la presente década, el tema de la diversidad sexual y de género en medio del conflicto armado comenzó a ganar renglones en el ámbito periodístico. Algunos títulos fueron: “Los 'paras' nos mataban por ser homosexuales" (Verdad Abierta, 2014), "El drama de los hombres violados en la guerra” (Escárraga, 2014), "Memoria histórica LGBT en Medellín” (CNMH, 2014). De igual forma, se encuentran otras reseñas en que sus títulos no hacen una alusión directa al tema, pero que a lo largo del texto relatan situaciones al respecto, algunas de ellas son: "Cuando las mujeres están en la mira” (León, 2014), "Los abortos perpetrados por los paras” (Jiménez, 2014), entre otras.

Estas notas periodísticas se caracterizan por ser crónicas que, aunque delatan la existencia del fenómeno, se quedan cortas para abarcar el trasfondo del tema. De manera que, sin desconocer que el medio periodístico ha sido fundamental para destacar la victimización de esta población, el carácter anecdótico y la poca rigurosidad investigativa de algunas publicaciones pueden suscitar interpretaciones vagas sobre la diversidad sexual y de género. 
Diversidad sexual y de género en el marco del conflicto armado en colombia. Algunas reflexiones para su estudio.

Por último, la academia también ha sido una instancia que ha contribuido en este proceso. Autores como José Fernando Serrano, Nancy Prada, Mauricio Albarracín, Pablo Bedoya, entre otros, han realizado acercamientos investigativos importantes en este campo de estudios, que todavía se encuentra en una etapa exploratoria en el país. De igual manera, no se puede olvidar la producción investigativa que han desarrollado las organizaciones sociales para sus informes de derechos humanos.

Todo este recorrido liderado por las organizaciones sociales, los medios de comunicación, la academia y por el propio Estado no ha sido sencillo, debido a las resistencias generadas por diferentes sectores del país. Uno de los hechos más destacados al respecto fue la centralidad que tuvo el enfoque de género en la contienda electoral del plebiscito del 2 de octubre de 2016, en el que cual hubo una estigmatización directa a la población LGBT.

\section{Reflexiones conceptuales, metodológicas y políticas para el estudio de la diversidad sexual y de género en el conflicto armado colombiano}

Los acercamientos investigativos sobre la diversidad sexual y de género en el marco del conflicto armado en Colombia aún se encuentran en una etapa exploratoria. Por tal motivo, es pertinente plantear algunas reflexiones que se podrían considerar para la puesta en marcha de este campo de estudios. Emprendiendo esta tarea, en las siguientes páginas se presentan una serie de observaciones: la denominación “LGBT” y la ruralidad, el acrónimo LGBT como una herramienta que encubre la interpretación de la victimización, la concentración de los análisis en la orientación e identidad sexual, la centralización en las víctimas que omite a los combatientes y el androcentrismo presente en los estudios al respecto.

\section{La sigla LGBT y la ruralidad}

Considerando que la mayoría de las víctimas del conflicto armado son de zonas rurales, es importante prestar atención a las maneras en que se aborda teórica, metodológica y epistemológicamente la diversidad sexual y de género en estos contextos. Como lo indican Serrano y Caribe Afirmativo (2016), hasta el momento la información sobre la situación de las personas LGBTI en zonas rurales es limitada. Los postulados bajo los cuales se han planteado los gay and lesbian studies, los LGBT studies, los queer studies y las premisas conceptuales de las organizaciones LGBT son predominantemente urbanas (Alabarracín y Rincón, 2013). Por esta razón, sus presupuestos no son necesariamente apropiados para abordar otras realidades como la ruralidad latinoamericana.

En primer orden, las personas con orientaciones sexuales e identidades de género no normativas en algunas regiones, y especialmente en las zonas rurales, no suelen reconocerse con una identidad tan definida (Albarracín y Rincón, 2013). Las propuestas teóricas anglosajonas 
parten de la "identidad" como elemento articulador de la sexualidad de los sujetos, premisa que no se cumple a cabalidad en algunos contextos latinoamericanos. Por ejemplo, algunos autores (Núñez, 1999; Urrea, Reyes y Botero, 2008; Gallego, 2010) han confirmado que para muchos varones de la región las prácticas sexuales no implican una orientación o una identidad sexual particular.

Segundo, las maneras de nombrar pueden ser distintas a las denominaciones "gay", "lesbiana”, "bisexual” o "transgénero" (CNMH, 2015). Cada una de estas nociones tiene un contenido histórico y político propio que, tal vez, no se relaciona con las realidades de las sexualidades e identidades de género no hegemónicas de los distintos territorios de la geografía nacional. Por ello, surgen otras maneras locales de denominarlas, como: marica, volteado, dañado, flor, guayaba, galleta, camionera, entre muchas otras. A pesar de que muchas de ellas surgieron para estigmatizar a aquellas personas, algunas se han convertido en investiduras bajo las cuales los sujetos emprenden ejercicios de autoafirmación.

En tercera instancia, el Estado, los organismos internacionales, los medios de comunicación y hasta los propios colectivos de derechos humanos no cuestionan la denominación "LGBT" para referirse a la diversidad sexual y de género de cualquier contexto. En este sentido, las políticas públicas y los programas de asistencia traen consigo maneras específicas de nombrar ciertas poblaciones o ciertos problemas sociales que eran descritos bajo otros calificativos o que simplemente ni siquiera eran reconocidos. Desde esta perspectiva, estas apuestas gubernamentales o multilaterales se convierten en mecanismos colonizadores que imponen marcos conceptuales foráneos, los cuales generan procesos de extrañamiento y de autoafirmación en los individuos. No obstante, estas plataformas burocráticas se han constituido en una oportunidad para el reconocimiento y la protección de los derechos de la diversidad sexual y de género en estos territorios.

\section{La sigla LGBT: una herramienta que ensombrece la victimización}

En los testimonios reunidos en los informes del CNMH, en los artículos de prensa y en los documentos judiciales regularmente aluden a una generalización: "víctima LGBT". Dicha denominación no se cuestiona ni siquiera cuando se tiene claro que el hecho hace alusión a una orientación sexual o identidad de género específica. Por tanto, surge una pregunta: ¿por qué no se nombra en singular?

En este sentido, la sigla LGBT se convierte en una herramienta conceptual que encubre las particularidades de los hechos victimizantes, restándole capacidad interpretativa a las labores judiciales, testimoniales y académicas. Diferentes autores e informes de derechos humanos han recalcado insistentemente que las violencias dirigidas contra esta población varían de acuerdo a cada caso (Prada, Herrera, Lozano y Ortiz, 2012; CNMH, 2015, 2017). Es decir, las agresiones 
Diversidad sexual y de género en el marco del conflicto armado en colombia. Algunas reflexiones para su estudio.

que sufren los hombres "gais", son distintas a las que reciben las personas trans o las mujeres lesbianas. Por esta razón, no se pueden reducir todos estos repertorios de victimización en un mismo vocablo. En el marco del conflicto armado este procedimiento, por ejemplo, reúne bajo una misma lógica de victimización la identidad de género, la orientación sexual y la expresión de género, nociones eminentemente distintas y que implican análisis específicos.

\section{Diversidad sexual: orientaciones, identidades y prácticas sexuales}

En general, los lesbian and gay studies, al igual que los LGBT studies se han concentrado en la orientación sexual y la identidad de género, dejando en un segundo plano otra categoría que está implícita en su campo de estudios: "las prácticas sexuales". Esta jerarquización tiene amplias repercusiones para la línea de investigación que estamos emprendiendo, pues las prácticas sexuales son un terreno propicio para el análisis, debido a que en un gran número de hechos victimizantes de orden sexual en los que están involucradas personas del mismo sexo biológico, los perpetradores o las víctimas no se identifican, necesariamente, con sexualidades e identidades no hegemónicas.

Sobre este asunto, List (2004) señala la necesidad de separar dos elementos: la práctica sexual y la identidad sexual. Esta distinción nos permite darnos cuenta de que se habla de dos cosas distintas y que los sujetos se reconocen o no como parte de un grupo sexual en la medida en que construyen una identidad con él. En otras palabras, las prácticas sexuales o las preferencias eróticas no siempre conllevan una identidad sexual. Este argumento es el postulado central del concepto "homoerotismo", sin embargo, esta noción no resulta pertinente para nuestro objeto de estudio debido a que algunas prácticas sexuales en contextos de guerra pueden implicar otros sentidos distintos al erotismo.

En los territorios en guerra, así como en los grupos armados, se presentan distintas prácticas sexuales entre personas del mismo sexo, en su mayoría entre hombres (CNMH, 2015, 2017). Algunas de ellas tienen objetivos militares, otras hacen parte de ritos grupales y otras con fines de placer. Uno de los casos más conocidos en términos victimizantes son los hombres violados en la guerra, sucesos que han sido denunciados en confrontaciones como las de Perú, Centroamérica y los Balcanes (Theidon, 2004; Sivakumaran, 2010), y que están empezando a ser reconocidos en el conflicto colombiano. Las agresiones sexuales contra hombres en contextos bélicos son predominantemente una afirmación del poder, en lugar de interpretarse como un medio para satisfacer un deseo sexual (Segato, 2003; Zawati, 2007; Franco, 2008).

En cuanto a lo ritual, se ha comprobado, por ejemplo, que en las guarniciones militares se recrean juegos sexuales entre compañeros, algunas veces como una oportunidad para demostrar su masculinidad y otras para satisfacer sus necesidades sexuales (Belkin y Levitt, 2001; Ortiz, 2004). Respecto a las prácticas en las que media el placer, por citar un caso en Colombia, se ha 
relatado cómo en algunas regiones algunos hombres gais o mujeres trans tuvieron relaciones sexuales con miembros de los grupos armados, sin que ese suceso desestabilizara de algún modo la heterosexualidad del combatiente (CNMH, 2015). Esta pluralidad de prácticas sexuales es una evidencia del potencial investigativo que representan las prácticas sexuales en la guerra, por ello se requieren herramientas conceptuales y metodológicas propicias para abordarlas. Como la plantea Viveros (2013):

\begin{abstract}
(...) plantear la pregunta sobre la pertinencia analítica y política de utilizar categorías como sexualidad, erotismo, género y amor para hablar de prácticas de grupos sociales que no se adecúan a los presupuestos sobre los cuales se fundaron dichas categorías es un reto para investigadoras e investigadores latinoamericanos que nos enfrentamos a realidades sociales muy complejas. (p. 41)
\end{abstract}

Partiendo del argumento anterior, tiene sentido cuestionarse: ¿qué reflexiones conlleva el hecho de que los mismos perpetradores de la guerra acudan a prácticas sexuales por fuera de la heterosexualidad? ¿Cómo plantearlas? ¿Cómo abordarlas?

\title{
Más allá de las víctimas: un estudio también sobre los combatientes
}

Los abordajes preliminares sobre diversidad sexual y de género en el conflicto armado se han concentrado en las víctimas. Esta disposición es producto de una apuesta institucional por parte del Estado colombiano, los organismos internacionales en cabeza de las Naciones Unidas y de las organizaciones no gubernamentales - ONG-. No obstante, la historia y la contingencia política del país permitirían aventurar otras aristas por medio de las cuales se indaguen las manifestaciones de las sexualidades e identidades de género no hegemónicas en medio de la guerra. Esta apuesta sería un ejercicio que enriquecería este campo de estudios y que complementaria la producción académica y jurídica sobre el conflicto armado en el país, incluso serían temas inéditos en el ámbito internacional. Algunos ejemplos podrían ser: ¿cómo se manifiesta la diversidad sexual y de género en los combatientes de los diferentes grupos armados? ¿Cómo se interpretan las prácticas sexuales entre personas del mismo sexo de acuerdo a las lógicas de cada grupo?

Hasta ahora, a nivel internacional las consideraciones de las sexualidades o prácticas sexuales por fuera del marco heterosexual en contextos bélicos se han concentrado en dos temas: en la violencia sexual contra los hombres (Sivakumaran, 2010) y en el reconocimiento de la diversidad sexual en las instituciones militares en varios Estados (Belkin y Levitt, 2001; Brown y Ayres, 2004). Frente al primer tema, los conflictos como los de Serbia, Congo, Ruanda e Irak con el caso de la cárcel de Abu Ghraib, han demostrado que los hombres también son receptores de violencias de orden sexual. En el caso colombiano se registran 1.224 hombres 
Diversidad sexual y de género en el marco del conflicto armado en colombia. Algunas reflexiones para su estudio.

víctimas, sin considerar aquellos que no lo han denunciado (Giraldo, 2018). Pero existe una misma lógica: la atención se dirige a las víctimas, por tanto, ¿cómo interpretamos la acción del perpetrador de estos casos de violencia? Pasando al segundo tema, debido a las luchas políticas de los movimientos sociales LGBT y de disidencias sexuales en las últimas décadas, se han reconocido los derechos de los hombres gais, mujeres lesbianas y personas trans que hacen parte de las fuerzas militares. Sin embargo, esta disposición institucional se ha convertido en una cortina de humo para el reconocimiento de otras situaciones en las guarniciones militares que implican prácticas sexuales abyectas, que lesionarían la legitimidad política y moral de la institución militar.

En el ámbito nacional, por su parte, existen algunos indicios que podrían catalogarse como una oportunidad para empezar a acercarse a estas realidades dentro de los grupos armados. Uno de ellos, por ejemplo, son las discusiones que se han tejido en la plataforma "LGBT por la paz" sobre la disposición de este colectivo para acoger a las personas combatientes que hacen parte de esta población. Otros rastros son algunos apartados del informe Aniquilar la diferencia. Lesbianas, gays, bisexuales y transgeneristas en el marco del conflicto armado (CNMH, 2015), en los que se comprueba la existencia de prácticas sexuales y relaciones sentimentales entre personas del mismo sexo al interior de las filas, antecedente que se convierte en un abrebocas para futuras investigaciones. Como lo admite el mismo informe en un apartado que lleva como título "Violencias heteronormativas entre filas":

\begin{abstract}
(...) este primer trabajo de memoria se enfocó en la reconstrucción de las violencias experimentadas por las personas víctimas del conflicto de modo que el proceso se concentró en las voces de estas víctimas y no en la de los combatientes. Sin embargo, algunas de estas personas habían sido parte de estructuras armadas lo que ha permitido realizar un acercamiento a estas violencias que ocurren en el interior de los grupos, no obstante, se requieren aún esfuerzos para profundizar en estas violencias a partir de las voces de integrantes de los grupos armados. (CNMH, 2015, p. 283)
\end{abstract}

En síntesis, existe un vacío investigativo de la diversidad sexual y de género al interior de los grupos armados tanto a nivel nacional como internacional. El escenario colombiano se presenta como una oportunidad para conocer otras perspectivas, tanto de los conflictos armados y las sexualidades por separado, como de sus intersecciones.

\title{
El androcentrismo en los estudios sobre diversidad sexual y de género
}

Por último, se plantea una discusión extra-teórica: el androcentrismo presente en los estudios sobre diversidad sexual y de género. Este campo del conocimiento tiene una cronología: en un primer momento, en los años 70, surgen los lesbian and gay studies; posteriormente, en 
la década de los 90 los LGBT studies; y finalmente los queer studies. Cada uno es producto de unas discusiones históricas, teóricas y epistemológicas particulares que sustentan sus objetos de estudio. En las tres etapas se destaca una condición: los temas de las mujeres han estado relegados a un segundo plano (Careaga, 2004; Barbosa y Facchini, 2009), a pesar de que en los últimos años se ha intentado remediar esta situación.

Este panorama, penosamente, se puede seguir presentando en la línea de estudio que se ha venido discutiendo a lo largo del texto. Ante la premisa de que "la guerra es de los hombres" existe una mayor atención en las identidades y prácticas sexuales en las que están involucrados hombres que transgreden el marco heteronormado. Una prueba de ello es que en el conjunto de hechos victimizantes hacia los hombres sobresalga la violencia sexual por encima de otros como el asesinato o la desaparición, o que se persiga con más vehemencia a los varones gais y a las mujeres trans, situaciones descritas por los diferentes informes y artículos de prensa (Lozano y Prada, 2012; Prada et al., 2012; CNMH, 2015, 2017). Estos antecedentes no se pueden convertir en una justificación arbitraria para que las investigaciones se concentren solo en estos casos. Es la oportunidad para que un campo de estudio que apenas está en emergencia no caiga en algunas carencias de líneas de investigación previas.

Aunque el campo de batalla sea representado tanto física como simbólicamente como un terreno de los "hombres", esta premisa no puede ser una excusa para no abordar los asuntos de las mujeres. Varios estudios se han encargado de reconocer la participación de las mujeres en sus diferentes facetas: como víctimas, como participantes de los grupos armados y como constructoras de paz (Wills y Gómez, 2006; Rayas, 2009; Ruta Pacífica de las Mujeres, 2013). Ahora, se puede emprender esta misma tarea integrando la dimensión de la diversidad sexual y de género.

\section{Conclusión}

\section{Los retos para la justicia transicional en clave de diversidad sexual y de género}

Para terminar, se presentarán una serie de reflexiones dirigidas a la justicia transicional. Este recurso político se ampara en cuatro principios: verdad, justicia, reparación y garantías de no repetición. Cada uno de los cuales tiene unas particularidades de acuerdo a la población que será atendida. En este caso, reflexionaremos en clave de diversidad sexual o de género.

\section{- Verdad}

De acuerdo a Cifuentes (2009): "Develar la forma como la dimensión de género penetra las estructuras y las lógicas del conflicto armado es fundamental para lograr una comprensión de fondo de este" (p. 129). En este sentido, el género y la sexualidad se convierten en elementos clave para los procesos de construcción de la "verdad" de las sociedades en transición. 
Diversidad sexual y de género en el marco del conflicto armado en colombia. Algunas reflexiones para su estudio.

Las garantías para la búsqueda de la verdad de las víctimas “LGBT” presentan algunos obstáculos. La diversidad sexual o de género no es fácilmente atribuible como en otras categorías del enfoque diferencial (pertenencia étnica, ciclo vital o discapacidad). La sexualidad de una persona puede quedar relegada a su ámbito privado, mientras su color de piel o su condición de diversidad funcional tienen un mayor grado de evidencia pública. Estas diferencias tienen una incidencia directa en los procesos jurídicos y políticos para construir la "verdad" de los hechos victimizantes, pues a mayor evidencia pública de la condición de vulnerabilidad, mayor es la probabilidad de atribución a ella y viceversa. Por ejemplo, las personas trans, en especial las mujeres, son reconocidas con menos trabas como víctimas "LGBT" debido a que su transgresión es evidente. La situación es distinta cuando son casos por orientación sexual ya que no siempre es un asunto notorio o divulgado por la persona. Por esta razón, la orientación sexual de las víctimas puede desaparecer estratégicamente en los testimonios de los victimarios -o de las propias víctimas- y de esa manera se afecta el esclarecimiento de lo ocurrido.

\section{- Justicia}

Como lo plantea Farr (2005) en su reflexión respecto a la Resolución 1325 del Consejo de Seguridad de las Naciones Unidas: "Alcanzar la justicia de género es tan central para la transformación social como cualquier otra forma de reparación en la posguerra” (p. 3). Sin embargo, el principio de “justicia” tiene varios retos para responder a las víctimas "LGBT". Para Serrano y Caribe Afirmativo (2016) este principio debe considerar:
(...) teniendo en cuenta las barreras históricas que han enfrentado estos grupos para acceder a la justicia, se deberán crear formas de investigación acordes al asunto, metodologías que reconozcan la variedad de voces de quienes conforman los sectores LGBTI y asegurar que los testimonios brindados no les re-victimicen. (p. 9)

A pesar de que las partes, tanto Gobierno nacional como las FARC-EP, hayan reconocido afectaciones diferenciales en estos sectores, el Estado colombiano no cuenta con una legislación específica que proteja la victimización de dicha población. Solo existe una ley general antidiscriminación (Ley 1482 de 2011) en la cual la orientación sexual y la identidad de género se suman a otras categorías. El Estado todavía está en deuda de una política pública nacional de diversidad sexual y de género. En este sentido, los Diálogos de La Habana se pueden convertir en un antecedente que evidencie la falta de mecanismos jurídicos concretos que atiendan la victimización de estas poblaciones, no solo en el marco del conflicto armado, sino también en la realidad nacional en su conjunto. Como lo señala Serrano (2013), los procesos de paz pueden ser una oportunidad para que se realicen cambios en las políticas y en los marcos legislativos desde los cuales se abordan los asuntos de diversidad sexual y de género (los casos de Irlanda del Norte y Sudáfrica - pos-apartheid-son un ejemplo de ello). 
Otros obstáculos para la búsqueda de la justicia son el subregistro (Lozano y Prada, 2012) y la tolerancia institucional de los organismos gubernamentales frente a la discriminación a la población "LGBT". Frente al subregistro, entre sus causas se encuentran: la homofobia estructural del país, la re-victimización, la falta de acceso a los organismos del Estado y la poca confianza en ellos. Estas circunstancias interfieren en la decisión de denunciar de la víctima y, por tanto, producen un desconocimiento de cifras verídicas de los casos de victimización. En cuanto a la tolerancia institucional, en muchos casos los mismos funcionarios y la misma institucionalidad son quienes reproducen estas violencias. Por este motivo, se requiere que la perspectiva de la diversidad sexual y de género se incorpore de una manera más eficiente en los programas de atención a víctimas y, en general, en todas las instancias del Estado, desde el orden nacional, hasta el departamental y local.

\section{- Reparación}

En términos de los Acuerdos de Paz de La Habana (Gobierno Nacional de Colombia, FARCEP y países garantes, 2016) la reparación comprende medidas de restitución, rehabilitación, indemnización y satisfacción tanto individuales como colectivas, siendo los distintos actores armados los responsables de brindar esas medidas materiales y simbólicas.

Teniendo en cuenta lo anterior, hay que considerar dos temas: primero, la población LGBT víctima del conflicto, a diferencia de otros sectores - mujeres, población afro o indígena-, no cuenta con el empoderamiento político suficiente para el reclamo de sus derechos, es decir, su capital político es limitado para emprender acciones de reparación (CNMH, 2015). A pesar de esta situación, la coyuntura política del país podría ser el terreno propicio para la consolidación de un movimiento LGBT con mayor incidencia política y más diseminado, ya que hasta ahora su campo de acción se concentra en las zonas urbanas y en algunas ciudades en específico (Bogotá, Cali, Barranquilla $)^{1}$, con poca presencia en los territorios más afectados por la violencia. De acuerdo a Serrano (2013), la construcción de paz permite la aparición de actores sociales que antes no eran considerados figuras legítimas del cambio social, y redefine su acción colectiva y su movilización. Por ejemplo, la organización social de las personas LGBT en El Salvador fue un fruto de la postguerra (Palevi, 2016). Sin embargo, como lo advierte França (2017) frente a los refugiados LGBT en España y Brasil, dichos movimientos no deben limitarse solamente al deseo de reconocimiento por parte del Estado, también deben articularse por medio de nuevas alianzas contra la violencia del Estado mismo.

\footnotetext{
${ }^{1}$ Se hace alusión a estas ciudades porque en ellas se localizan las organizaciones Colombia Diversa, Santamaría Fundación y Caribe Afirmativo que, además de ser las más importantes en el país, han emprendido trabajos reconocidos nacionalmente con población víctima del conflicto armado. En los últimos años, en algunos territorios han surgido algunas iniciativas como el Colectivo LGBT de los Montes de María, la Mesa LGBT de la Comuna 8 de Medellín, Chaparral Diversa en el Tolima, entre otras, que han emprendido estos esfuerzos, pero sus alcances son limitados debido a que disponen de menores capitales económicos, sociales y políticos.
} 
Diversidad sexual y de género en el marco del conflicto armado en colombia. Algunas reflexiones para su estudio.

Segundo, los distintos actores armados asumen de manera distinta la agenda LGBT: para el Estado es un tema en el que ya ha incursionado debido a las discusiones políticas y jurídicas frente a esta población sucedidas en la última década en el país, mientras que para las FARC-EP es un asunto que había evitado en sus filas (CNMH, 2015), por lo que es un tema sobre el cual tienen poca facultad en términos políticos, aunque en las premisas del "feminismo insurgente" propuesto por las excombatientes se involucran los asuntos de diversidad sexual y de género. Al respecto, Serrano y Caribe Afirmativo (2016) advierten la necesidad de que la reintegración social de los combatientes vaya acompañada de procesos que transformen imaginarios y formas de relación que justificaron las violencias contra personas y organizaciones de los sectores LGBTI. Esta asimetría entre los actores armados tendría repercusiones en las acciones de reparación de las víctimas. Por ello, los diferentes actores deben tener una apertura a la incorporación de la perspectiva de diversidad sexual y de género, puesto que la inclusión de este enfoque podría ampliar los límites del proceso de construcción de paz en Colombia (Serrano, 2013).

\section{Garantías de no repetición}

Para terminar, en los últimos años en Colombia y, en general, en América Latina se ha venido presentando un aumento de las manifestaciones de homofobia generadas, principalmente, por la mal llamada "ideología de género" (Barrientos, 2015; Efrem, 2016). Luego de que la década de los 2000 fuera un periodo de grandes avances para la agenda "LGBT", en los últimos años se han evidenciado algunos retrocesos. Estas discusiones se han generado por el matrimonio y la adopción igualitaria, y en los últimos años por algunos proyectos de educación sexual planteados por organismos del Estado que, según algunos sectores, atentan contra la moral y la familia. Lo anterior ha generado la aparición de amplios sectores políticos y religiosos con fuertes discursos homofóbicos. Algunas cifras del documento Entre el miedo y la resistencia. Informe de Derechos Humanos de personas LGBT en Colombia 2016, liderado por Colombia Diversa, Caribe Afirmativo y Santamaría Fundación (2016), demuestran esta situación: en el 2015 fueron asesinadas 108 personas, en el 2016 fueron asesinadas 7 personas defensoras de derechos humanos, y se registraron 256 agresiones físicas y 77 casos de violencia policial contra esta población.

En palabras de Salcedo (2013):

(...) la perspectiva de género debe ser el punto de partida para la elaboración de medidas que busquen garantizar los derechos de las víctimas; el hecho de nombrarlas, reconocerlas, apoyarlas y fortalecerlas permite reparar las opresiones estructurales y las violaciones a sus derechos que tengan lugar en el marco del conflicto armado. (p. 148) 
Sin embargo, ante este panorama de homofobia estructural es difícil suponer unas garantías de no repetición a las víctimas "LGBT" del conflicto armado. Tal vez ya no serán victimizadas por los grupos armados, pero seguirán en un entorno social que las ubica material y simbólicamente en lo abyecto. Por lo tanto, como lo señala el CNMH (2015), se podría plantear un continuum de violencias frente a esta población que perdura antes, durante y después del conflicto armado.

En este sentido, como lo advierte Serrano (2013), los periodos de postguerra pueden generar una exageración de las representaciones sobre el género y la sexualidad. En específico, la agenda de género casi siempre está implicada en los discursos nacionalistas formulados en ciertas coyunturas políticas, debido a que se entiende como un dispositivo fundamental para lograr el orden de la nación (Fassin, 2009), argumento que recuerda el carácter generizado de las naciones y del nacionalismo (Nagel, 2003). Por ello, el género - y de paso la sexualidad-no deben seguir quedando relegados de las discusiones políticas en torno a la construcción de paz.

\section{Referencias}

Albarracín, M. y Rincón, J. C. (2013). De las víctimas invisibles a las víctimas dignificadas: los retos del enfoque diferencial para la población LGBTI en la Ley de Víctimas. Revista de Derecho Público, 31, 1-31.

Barbosa, R. y Facchini, R. (2009). Acesso a cuidados relativos à saúde sexual entre mulheres que fazem sexo com mulheres em São Paulo, Brasil. Cadernos Saúde Pública, 25 (2), 291 300 .

Barrientos, J. (2015). Violencia homofóbica en América Latina y Chile. Santiago de Chile, Chile: Ediciones El Buen Aire.

Belkin, A. \& Levitt, M. (2001). Homosexuality and the Israel Defense Forces: Did Lifting the Gay Ban Undermine Military Performance? Armed Forces and Society, 27 (4), 541-565.

Brown, J. \& Ayres, I. (2004). The Inclusive Command: Voluntary integration of sexual minorities into the US military. Michigan Law Review, 103 (150), 150-188.

Careaga, G. (2004). Relaciones entre mujeres. En C. Cáceres., T. Frasca., M. Pecheny. y V. Terto. (Eds.), Ciudadanía sexual en América Latina: Abriendo el debate (pp. 261-272). Lima, Perú: Universidad Peruana Cayetano Heredia.

Centro Nacional de Memoria Histórica -CNMH-. (2013). Desafíos para la Reintegración: Enfoques de género, edad y etnia. Bogotá, Colombia: CNMH, USAID, OIM.

Centro Nacional de Memoria Histórica-CNMH-. (2014). Memoria histórica LGBT en Medellín. Noticias CNMH. Recuperado de http: / / www.centrodememoriahistorica.gov.co/noticias/ noticias-cmh/memoria-historica-lgbt-en-medellin 
Diversidad sexual y de género en el marco del conflicto armado en colombia. Algunas reflexiones para su estudio.

Centro Nacional de Memoria Histórica-CNMH-. (2015). Aniquilar la diferencia. Lesbianas, gays, bisexuales y transgeneristas en el marco del conflicto armado colombiano. Bogotá, Colombia: CNMH - UARIV - USAID - OIM.

Centro Nacional de Memoria Histórica-CNMH-. (2017). La guerra inscrita en el cuerpo. Informe nacional de violencia sexual en el conflicto armado. Bogotá, Colombia: CNMH.

Cifuentes, M. R. (2009). La investigación sobre género y conflicto armado. Revista Eleuthera, 3 (1), 127-164.

Colombia Diversa, Caribe Afirmativo y Santamaría Fundación. (2016). Entre el miedo y la resistencia. Informe de Derechos Humanos de personas LGBT en Colombia 2016. Bogotá, Colombia: Colombia Diversa.

Efrem, R. (2016). Corpos brutalizados: conflitos e materializações nas mortes de LGBT. Cadernos Pagu, 46, 311-340.

Escárraga, T. (6 de septiembre de 2014). El drama de los hombres violados en la guerra. El Tiempo. Recuperado de http://www.eltiempo.com/archivo/documento/CMS14496395

Farr, V. (2005). La desmilitarización con perspectiva de género como herramienta para la construcción de la paz. Cuadernos INER, Serie Traducciones, 2, 1-40.

Fassin, E. (2009). Género, sexualidades y política democrática. Ciudad de México, México: UNAMPUEG.

França, I. L. (2017). "Refugiados LGBTI": direitos e narrativas entrecruzando gênero, sexualidade e violência. Cadernos Pagu, 50, e17506.

Franco, J. (2008). La violación: Un arma de guerra. Debate Feminista, 37, 16 -33.

Gallego, G. (2010). Demografía de lo otro. Biografías sexuales y trayectorias de emparejamiento entre varones en la Ciudad de México. Ciudad de México, México: El Colegio de México.

Giraldo, S. (2018). Hombres víctimas de los conflictos armados. Algunas reflexiones a partir del caso colombiano. En F. Lanuza. (Coord.), Masculinidades, delincuencia organizada y violencia social en México (pp. 78-92). Querétaro, México: Universidad Autónoma de Querétaro.

Gobierno Nacional de Colombia, FARC-EP y países garantes. (2016). Acuerdo final para la terminación del conflicto y la construcción de una paz estable y duradera. Recuperado de http: / / www.altocomisionadoparalapaz.gov.co/procesos-y conversaciones/Documentos\%20 compartidos/24-11-2016NuevoAcuerdoFinal.pdf

Goldstein, J. (2003). War and gender: How gender shapes the war system and vice versa. Cambridge, England: Cambridge University Press. 
Jiménez, J. S. (29 de noviembre de 2014). Los abortos perpetrados por los paras. El Espectador. Recuperado de https://www.elespectador.com/noticias/judicial/los-abortosperpetrados-los-paras-articulo-530479

León, J. (16 de diciembre de 2014). Cuando las mujeres están en la mira. La Silla Vacía. Recuperado de http://lasillavacia.com/historia/historia-mujeres-aterrizaje-de-lapaz-49305

Ley 975 de 2005 (25 de julio). Por la cual se dictan disposiciones para la reincorporación de miembros de grupos armados organizados al margen de la ley, que contribuyan de manera efectiva a la consecución de la paz nacional y se dictan otras disposiciones para acuerdos humanitarios. Diario Oficial No. 45.980.

Ley 1448 de 2011 (10 de junio). Por la cual se dictan medidas de atención, asistencia y reparación integral a las víctimas del conflicto armado interno y se dictan otras disposiciones. Diario Oficial No. 48.096.

Ley 1482 de 2011 (1 de diciembre). Por medio de la cual se modifica el Código Penal y se establecen otras disposiciones. Diario Oficial No. 48.270.

List, M. (2004). Masculinidades diversas. Revista de Estudios de Género La Ventana, 20, 101-117.

Lozano, L. T. y Prada, N. (2012). Mujeres trans y conflicto armado en Colombia: afectaciones específicas y retos para la implementación de la ley de víctimas. En B. I. Arteaga., D. A. Walteros. y O. D. Andrade. (Coords.), Identidades, enfoque diferencial y construcción de paz (pp. 75-98). Bogotá, Colombia: Universidad Jorge Tadeo Lozano.

Nagel, J. (2003). Fronteras etnosexuales en zonas de guerra. Nómadas, 19, 188-199.

Núñez, G. (1999). Sexo entre varones. Poder y resistencia. Sonora, México: PUEG-UNAM/El Colegio de Sonora/Miguel Ángel Porrúa.

ONU. (2000). Resolución 1325 del Consejo de Seguridad de las Naciones Unidas: "Adopción de una perspectiva de género que incluye las necesidades especiales de las mujeres y las niñas durante la repatriación y reasentamiento, la rehabilitación, la reintegración y la reconstrucción post-conflicto”. Recuperado de http://www.acnur.org/t3/fileadmin/Documentos/BDL/2006/1759. pdf

Ordóñez, J. P. (1996). No Human Being is Disposable: Social Cleansing, Human Rights and Sexual Orientation in Colombia. Washington: IGLHRC.

Ortiz, C. (2004). Reflexiones en torno a la homosexualidad y fuerzas armadas. Fuerzas Armadas y Sociedad, 18 (3-4), 259-270.

Palevi, A. (2016). Travestis, marimachas y maricones: el camino del arcoíris en El Salvador. Revista Punto Género, 6, 93-112. 
Diversidad sexual y de género en el marco del conflicto armado en colombia. Algunas reflexiones para su estudio.

Prada, N., Herrera, S., Lozano, L. T. y Ortiz, A. N. (2012). “ ¡A mí me sacaron volada de allá!”. Relatos de mujeres trans desplazadas forzosamente hacia Bogotá. Bogotá, Colombia: Alcaldía Mayor de Bogotá y Universidad Nacional de Colombia.

Rayas, L. (2009). Armadas: un análisis de género desde el cuerpo de las mujeres combatientes. Ciudad de México, México: Colegio de México.

Ruta Pacífica de las Mujeres. (2013). La verdad de las mujeres víctimas del conflicto armado en Colombia. Bogotá, Colombia: Ruta Pacífica de las Mujeres.

Salcedo, D. M. (2013). Género, derechos de las víctimas y justicia transicional: Retos en Colombia. Revista de Paz y Conflictos, 6, 124-151.

Segato, R. (2003). Las estructuras elementales de la violencia. Ensayos sobre género entre la antropología, el psicoanálisis y los derechos humanos. Buenos Aires, Argentina: Universidad Nacional de Quilmes.

Serrano, J. F. (2004). Queering conflict:The invisibility of gender and sexual diversity in peace building (Master's Thesis). University of Bradford, Bradford, England.

Serrano, J. F. (2013). Agenciamiento e (in)visibilidad de la diversidad sexual y de género en la construcción de paz. En J. F. Serrano. y A. Baird. (Eds.), Paz, paso a paso. Una mirada a los conflictos colombianos desde los estudios de paz (pp. 53-78). Bogotá, Colombia: CINEP, Universidad Javeriana.

Serrano, J. F. y Caribe Afirmativo. (2016). Oportunidades y retos de la inclusión del enfoque de género y diversidad sexual en los acuerdos de la Mesa de conversaciones. Recuperado de http: / / library.fes.de/pdf-files/bueros/kolumbien/12782.pdf

Sivakumaran, S. (2010). Del dicho al hecho: la ONU y la violencia sexual contra hombres y niños durante conflictos armados. International Review of the Red Cross, 877, 1-20.

Theidon, K. (2004). Entre prójimos: el conflicto armado interno y la política de la reconciliación en el Perú. Lima, Perú: Instituto de Estudios Peruanos.

Unidad para la Atención y Reparación Integral a las Víctimas. (2014). Informe víctimas del conflicto armado interno con orientaciones sexuales diversas. Recuperado de https://rni. unidadvictimas.gov.co/sites/default/files/Documentos/orientaciones\%20sexuales\%20 diversas.pdf

Urrea F., Reyes, J. y Botero,W. (2008). Tensiones en la construcción de identidades de hombres negros homosexuales en Cali. En P. Wade., F. Urrea. y M. Viveros. (Eds.), Raza, etnicidad y sexualidades. Ciudadanía y multiculturalismo en América Latina (pp. 279-316). Bogotá, Colombia: Universidad Nacional de Colombia. 
USAID, Organización Internacional para las Migraciones y Unidad para la Atención y Reparación Integral a las Víctimas. (2015). Enfoque de orientaciones sexuales e identidades de género. Recuperado de https://www.unidadvictimas.gov.co/sites/default/files/ documentosbiblioteca/sexualidad.PDF

Verdad Abierta. (25 de noviembre del 2014). "Los 'paras' nos mataban por homosexuales". Verdad Abierta. Recuperado de https://verdadabierta.com/los-paras-nos-mataban-porser-homosexuales/

Viveros, M. (2013). Alteridad, género, sexualidad y afectos: reflexiones a partir de una experiencia investigativa en Colombia. Cadernos Pagu, 41, 41-52.

Wills, M. y Gómez, D. (2006). Los movimientos sociales de mujeres (1970-2005). Innovaciones, estancamientos y nuevas apuestas. En F. Leal. (Ed.), En la encrucijada. Colombia en el siglo XXI (pp. 291-321). Bogotá, Colombia: Norma.

Zawati, H. M. (2007). Impunity or Immunity: Wartime Male Rape and Sexual Torture as a Crime against Humanity. Journal on Rehabilitation of Torture Victims and Prevention of Torture, 17 (1), 27-47. 